OPEN ACCESS

Edited by:

John Perfect,

Duke University, United States

Reviewed by:

Popchai Ngamskulrungroj,

Mahidol University, Thailand

Methee Chayakulkeeree,

Mahidol University, Thailand

*Correspondence:

Kirsten Nielsen

knielsen@umn.edu

Specialty section: This article was submitted to

Fungal Pathogenesis,

a section of the journal

Frontiers in Cellular and

Infection Microbiology

Received: 14 April 2021

Accepted: 10 May 2021

Published: 23 June 2021

Citation:

Gerlach ES, Altamirano S, Yoder JM,

Luggya TS, Akampurira A, Meya DB,

Boulware DR, Rhein $\mathrm{J}$ and Nielsen $\mathrm{K}$ (2021) ATI-2307 Exhibits Equivalent

Antifungal Activity in Cryptococcus

neoformans Clinical Isolates With High

and Low Fluconazole $1 C_{50}$

Front. Cell. Infect. Microbiol. 11:695240.

do: 10.3389/fcimb.2021.695240

\section{ATI-2307 Exhibits Equivalent Antifungal Activity in Cryptococcus neoformans Clinical Isolates With High and Low Fluconazole $\mathbf{I C}_{50}$}

\author{
Elliot S. Gerlach ${ }^{1}$, Sophie Altamirano ${ }^{1}$, J. Marina Yoder ${ }^{1}$, Tony S. Luggya ${ }^{2}$, \\ Andrew Akampurira ${ }^{2}$, David B. Meya ${ }^{2}$, David R. Boulware ${ }^{3}$, Joshua Rhein ${ }^{3}$ \\ and Kirsten Nielsen ${ }^{1 *}$ \\ ${ }^{1}$ Department of Microbiology and Immunology, University of Minnesota, Minneapolis, MN, United States, ${ }^{2}$ Infectious \\ Diseases Institute, College of Health Sciences, Makerere University, Kampala, Uganda, ${ }^{3}$ Division of Infectious Diseases \\ and International Medicine, Department of Medicine, University of Minnesota, Minneapolis, MN, United States
}

Half maximal inhibitory concentrations $\left(\mathrm{IC}_{50}\right)$ to the experimental drug ATI-2307 and complete inhibition $\left(\mathrm{IC}_{90}\right)$ of the common clinically used antifungal drug amphotericin $\mathrm{B}$ were determined by microbroth dilution assay for a collection of 69 clinical isolates of Cryptococcus neoformans from Uganda that had high fluconazole $\mathrm{IC}_{50}$ values. The majority of the clinical isolates tested had fluconazole $\mathrm{IC}_{50}$ at or above $8 \mu \mathrm{g} / \mathrm{mL}$, but were susceptible to both amphotericin B ( $\left.\mathrm{IC}_{90} \leq 1 \mu \mathrm{g} / \mathrm{mL}\right)$ and ATI-2307 (IC50 $\leq 0.0312$ $\mu \mathrm{g} / \mathrm{mL})$. No correlation between increased fluconazole minimum inhibitory concentration (MIC) and ATI-2307 or amphotericin B MIC was observed, suggesting that the cellular changes impacting fluconazole susceptibility did not impact the effectiveness of ATI-2307. Our results suggest that ATI-2307 is a promising new antifungal drug for use in the context of high fluconazole or other antifungal drug MICs and/or in combination drug therapy regimens.

Keywords: ATI-2307, Cryptococcus, azole, antifungal, susceptibility, resistance, IC ${ }_{50}$, T-2307

\section{INTRODUCTION}

Cryptococcus neoformans is an environmentally acquired pathogenic yeast that causes the disease cryptococcal meningitis. Disease is typically observed in immunocompromised individuals, particularly those with advanced HIV/AIDS, and thus is a leading cause of mortality in subSaharan Africa (Park et al., 2009; Rajasingham et al., 2017; Hurtado et al., 2019). For cryptococcal meningitis, the small repertoire of antifungal drugs remains a critical limitation. The location of the 
infection in the central nervous system, where the blood-brain barrier complicates or limits drug dissemination, further reduces the antifungal drugs that can be used successfully for cryptococcal meningitis therapy (Felton et al., 2014; Roemer and Krysan, 2014). In addition, because many cryptococcal meningitis patients are immunocompromised and thus often receiving other drug treatments, drug-drug interactions must be considered (Vadlapatla et al., 2014). The most effective antifungal drug for treatment of cryptococcal meningitis, amphotericin B, is known for its potential to cause significant side effects and need for intravenous administration (Klepser, 2011).

There is increasing documentation of differences in antifungal drug MICs in C. neoformans isolates from cryptococcal meningitis patients (Pfaller et al., 1998; Assing et al., 2003; Archibald et al., 2004; Mdodo et al., 2011). These differences are best documented in the azole drug fluconazole where $\mathrm{IC}_{50}$ values range from 0.25 to $>256 \mu \mathrm{g} / \mathrm{mL}$ (Pfaller et al., 2011; Chen et al., 2015; Smith et al., 2015; Bongomin et al., 2018; Chesdachai et al., 2019; Naicker et al., 2020). To date, clinical breakpoints (CBPs) are not clearly established within the field. Studies to identify epidemiologic cut-off values show $\mathrm{IC}_{50}$ of 4 or $8 \mu \mathrm{g} / \mathrm{mL}$, but there is significant variability depending upon geographic region and across time (Mdodo et al., 2011; Pfaller et al., 2011; Smith et al., 2015; Pharkjaksu et al., 2020). Thus, clinical drug susceptibility and resistance are challenging to define in C. neoformans and instead strains are often classified based on high or low $\mathrm{IC}_{50} / \mathrm{IC}_{90}$ values upon in vitro $\mathrm{MIC}$ assay.

Fluconazole is a broad-spectrum antifungal commonly used for the treatment of other fungal infections observed in immunocompromised patients, such as mucosal candidiasis and fungal skin infections, and is commonly prescribed to HIV-seropositive patients. This previous fluconazole exposure for other indications may be causing the fluctuations in fluconazole MIC observed in cryptococcal meningitis patients (Naicker et al., 2020). Alternatively, azole fungicides are also commonly used in agriculture, and azole cross-resistance is well documented in other fungal species (Verweij et al., 2009). Given that $C$. neoformans is environmentally acquired, this agricultural azole use could also be driving changes in fluconazole MIC of clinical strains (Smith et al., 2015).

Fluconazole can be used in combination therapy with amphotericin B and is the drug of choice for consolidation therapy after initial amphotericin B treatment (Molloy et al., 2018). Currently, fluconazole still has a major role in prevention of meningitis in the preemptive treatment of persons with early disseminated cryptococcal infection, termed cryptococcal antigenemia (Meya et al., 2010; Nalintya et al., 2018). Fluconazole targets the ergosterol biosynthesis pathway, disrupting fungal cell membrane structure and formation. Given the increasing recognition of fluconazole resistance within C. neoformans clinical isolates, development of additional drug treatments, with different modes of action, is needed.

ATI-2307 (formerly T-2307 at FUJIFILM Toyama Chemical Co. Ltd) is a pentamidine-like compound with antifungal activity currently under development at Appili Therapeutics, Inc. ATI-2307 has a broad spectrum of activity against many fungal pathogens including Candida spp, Aspergillus spp., and C. neoformans. The mode of action of ATI-2307 is different from the azoles; ATI-2307 acts via selectively inhibiting yeast mitochondrial respiratory chain complexes III and IV (Mitsuyama et al., 2008; Shibata et al., 2012; Yamashita et al., 2019). Here we tested the antifungal activity of ATI-2307 on isolates of $C$. neoformans with high fluconazole $\mathrm{IC}_{50}$ sampled from cryptococcal meningitis patients enrolled in multiple clinical trials in Uganda. This analysis was performed to demonstrate that ATI-2307 maintains activity in a diverse set of $C$. neoformans isolates common to the patient population for which the drug may be developed, and to determine if there was a correlation between elevated fluconazole MIC and increased ATI-2307 MIC.

\section{MATERIALS AND METHODS}

\section{Drugs and Dilutions}

4 - $\{3$ - [1-(3- $\{4$ - [amino(imino)-methyl]phenoxy $\}$ propyl) piperidin-4-yl]propoxy\}benzamidine (T-2307, ATI-2307) was provided by Appili Therapeutics, Inc. (Nova Scotia, Canada) as a trihydrochloride pentahydrate salt. Fluconazole and amphotericin B were purchased from Sigma-Aldrich (St. Louis, MO). A $10 \mathrm{mg} / \mathrm{mL}$ stock solution of ATI-2307 was prepared in sterile water then further diluted to a working stock solution of $59.904 \mu \mathrm{g} / \mathrm{mL}$ free base ATI-2307. Final microbroth dilution assay concentrations were determined from a value of $68.7 \%$ free base to salt, with the molecular weight of each being $437.59 \mathrm{~g} / \mathrm{mol}$ and $637.04 \mathrm{~g} / \mathrm{mol}$, respectively. A $50 \mathrm{mg} / \mathrm{mL}$ stock solution of fluconazole was prepared in DMSO. Amphotericin B was acquired pre-diluted to $250 \mu \mathrm{g} / \mathrm{mL}$ in sterile water. The ATI-2307 test concentrations ranged from $0.0004875-0.2496 \mu \mathrm{g} / \mathrm{mL}$, the fluconazole test concentrations ranged from $0.25-128 \mu \mathrm{g} / \mathrm{mL}$, and the amphotericin B test concentrations ranged from 0.0078125 $-4 \mu \mathrm{g} / \mathrm{mL}$.

\section{Inoculum Preparation}

Clinical isolates of $C$. neoformans with high, intermediate, and low levels of fluconazole susceptibility, obtained as part of the ASTRO trials (Rhein et al., 2016; Rhein et al., 2019) or COAT trial (Boulware et al., 2014) were plated onto yeast-peptonedextrose (YPD) plates containing $0.04 \mathrm{mg} / \mathrm{mL}$ chloramphenicol and incubated at $30^{\circ} \mathrm{C}$ for 48 hours. Overnight cultures were subsequently prepared in YPD broth containing $10 \mu \mathrm{g} / \mathrm{mL}$ chloramphenicol and incubated at $30^{\circ} \mathrm{C}$ with shaking. Cells were centrifuged and washed 3 times with sterile water, resuspended, and a 1:100 dilution was prepared for quantification via hemocytometer. The final inoculum of each isolate for the microbroth dilution MIC assay was prepared to the subsequent EUCAST specifications (Arendrup et al., 2017) in sterile water. 


\section{Microbroth Dilution MIC Assays}

Broth microdilution assays were carried out according to the EUCAST protocol following the subsection "Cryptococcus spp" recommendations, using a 2\% glucose RPMI-1640 medium (Sigma R8755) with a final inoculum concentration of $0.5 \mathrm{x}$ $10^{5}-2.5 \times 10^{5}$ (Arendrup et al., 2017). The RPMI-1640 used contained no phenol red indicator. Immediately following inoculation, optical density at 600nm wavelength (OD600) measurements were obtained on a Biotek Synergy H1 Hybrid reader (Winooski, VT). Plates were then incubated 72 hours at $37^{\circ} \mathrm{C}$, and a second OD600 measured. The $\mathrm{IC}_{50}$ or $\mathrm{IC}_{90}$ for each strain was determined based on analysis of the well turbidity measurements using the OD600, as described in Smith et al. (2015) with $\mathrm{IC}_{50}$ defined as the drug concentration at which growth was limited to 50 percent of baseline growth in the absence of drug and $\mathrm{IC}_{90}$ defined as the drug concentration at which growth was limited to 10 percent of baseline growth (calibrated as no visible growth) in the absence of drug. KN99 $\alpha$ (Nielsen et al., 2003), with a known fluconazole $\mathrm{IC}_{50}$ of $2 \mu \mathrm{g} / \mathrm{mL}$ and amphotericin B $\mathrm{IC}_{90}$ of $0.5 \mu \mathrm{g} / \mathrm{mL}$ (Smith et al., 2015) and ATI-2307 IC $_{50}$ of $0.0078 \mu \mathrm{g} / \mathrm{mL}$ (this study), was included as an inter-assay calibration reference in every MIC plate to verify accuracy across MIC plates.

\section{Analysis}

Association between fluconazole, amphotericin B, and ATI2307 susceptibility was compared with generalized linear regression models using GraphPad Prism version 9.0.2 (San Diego, CA).

\section{RESULTS}

We analyzed 69 C. neoformans clinical isolates from Uganda with varying levels of fluconazole $\mathrm{IC}_{50}$ from $<8 \mu \mathrm{g} / \mathrm{mL}(\mathrm{n}=6)$, $8 \mu \mathrm{g} / \mathrm{mL}(\mathrm{n}=28), 16 \mu \mathrm{g} / \mathrm{mL}(\mathrm{n}=22)$, and 32 or $64 \mu \mathrm{g} / \mathrm{mL}(\mathrm{n}=9)$ (Table 1). All 68 isolates had low amphotericin $\mathrm{B} \mathrm{IC}_{90}$ of $0.5 \mu \mathrm{g} /$ $\mathrm{mL}(\mathrm{n}=16), 1.0 \mu \mathrm{g} / \mathrm{mL}(\mathrm{n}=52)$, and $2.0 \mu \mathrm{g} / \mathrm{mL}(\mathrm{n}=1)$. Similar to previous reports (Casadevall et al., 1993; Perea and Patterson, 2002), we observed no correlation between fluconazole $\mathrm{IC}_{50}$ and amphotericin $\mathrm{B} \mathrm{IC}_{90}$ (Figure 1A, rho=0.2049, $P=0.0912$ ). All 69 isolates also fell within the previously established ATI-
$2307 \mathrm{IC}_{50}$ range of $0.0078-0.0624 \mu \mathrm{g} / \mathrm{mL}$ (Mitsuyama et al., 2008) for C. neoformans strains (Table 1). Again, we observed no correlation between fluconazole $\mathrm{IC}_{50}$ and ATI-2307 $\mathrm{IC}_{50}$ (Figure 1B, rho $=-0.1690, P=0.1650$ ). In addition, we showed no correlation between amphotericin $\mathrm{B} \mathrm{IC}_{90}$ and ATI-2307 $\mathrm{IC}_{50}$ values (Figure 1C, rho=0.0125, $P=0.9190$ ). Finally, we determined $\mathrm{MIC}_{50}$ and $\mathrm{MIC}_{90}$ values for ATI-2307 and amphotericin B, respectively, and showed they remain equivalent when the strains were analyzed based on fluconazole $\mathrm{IC}_{50}$ values (Table 2).

\section{DISCUSSION}

The in vitro EUCAST broth microdilution assay $\mathrm{IC}_{50}$ measurements for $C$. neoformans clinical isolates showed no correlation between high fluconazole $\mathrm{IC}_{50}$ and activity of the experimental antifungal drug ATI-2307 or amphotericin B. All the Ugandan clinical isolates tested had $\mathrm{IC}_{50}$ values for ATI-2307 that were below the $0.0624 \mu \mathrm{g} / \mathrm{mL}$ threshold previously identified in C. neoformans by Mitsuyama et al. (2008), irrespective of fluconazole sensitivity. While we only analyzed strains from Uganda and differences in fluconazole resistance are observed in populations from across the globe, given the completely different mode of action of ATI-2307 it is likely that our results showing no association between ATI-2301 and fluconazole resistance will be representative for C. neoformans. Consistent with the in vitro activity, similar drug concentrations are effective in murine models of C. neoformans (Mitsuyama et al., 2008) and C. gattii (Nishikawa et al., 2017), suggesting that therapeutic levels of ATI-2307 against Cryptococcus species can be achieved in vivo.

A similar study with the novel azole-derivative VT-1129 showed a positive correlation with increasing fluconazole $\mathrm{IC}_{50}$, although the increase in VT-1129 $\mathrm{IC}_{50}$ was not biologically significant due to the enhanced potency of VT-1129 compared to fluconazole (Nielsen et al., 2017). In contrast, and as expected due to the different modes of action of ATI-2307 and fluconazole, our study shows no reduction in ATI-2307 potency in the high fluconazole $\mathrm{IC}_{50}$ clinical isolates. As such, ATI-2307 may be a good candidate to pursue for frontline therapy in areas where high fluconazole MICs are known to occur, or as second line therapy when drug treatment has failed due to fluconazole resistance.

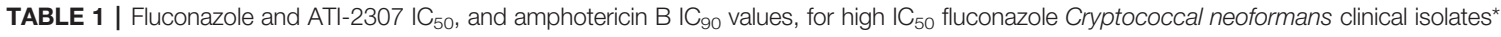

\begin{tabular}{|c|c|c|c|c|c|c|c|c|}
\hline \multicolumn{9}{|c|}{ Low $^{+}$} \\
\hline Fluconazole $\mathrm{IC}_{50}(\mu \mathrm{g} / \mathrm{mL})$ & 0.5 & 1 & 2 & 4 & 8 & 16 & 32 & 64 \\
\hline Isolates N (cumulative \%) & $2(3)$ & $3(7)$ & $1(9)$ & $0(9)$ & $28(49)$ & $26(87)$ & $6(96)$ & $3(100)$ \\
\hline $\begin{array}{l}\text { Amphotericin } B I_{90}(\mu \mathrm{g} / \mathrm{mL}) \\
\text { Isolates } \mathrm{N} \text { (cumulative } \%)\end{array}$ & $\begin{array}{c}0.5 \\
16(23)\end{array}$ & $\begin{array}{c}1.0 \\
52(99)\end{array}$ & $\begin{array}{c}2.0 \\
3(100)\end{array}$ & & & & & \\
\hline $\begin{array}{l}\text { ATI-2307 IC } 50(\mu \mathrm{g} / \mathrm{mL}) \\
\text { Isolates } \mathrm{N} \text { (cumulative \%) }\end{array}$ & $\begin{array}{l}0.0078 \\
26(38)\end{array}$ & $\begin{array}{l}0.0156 \\
39(94)\end{array}$ & $\begin{array}{r}0.0312 \\
3(99)\end{array}$ & $\begin{array}{l}0.0624 \\
1(100)\end{array}$ & & & & \\
\hline
\end{tabular}

$1 C_{50}$ and $I C_{90}$ values were determined using the EUCAST microbroth dilution method (Arendrup et al., 2017).

${ }^{*} A$ total of 69 isolates were screened, and data are presented as the number (cumulative percentage) of isolates with growth inhibition at (or below, for cumulative percentage) the indicated drug concentration.

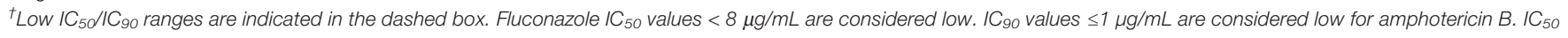
values $\leq 0.0624 \mu \mathrm{g} / \mathrm{mL}$ are susceptible to ATI-2307 based on murine studies (Mitsuyama et al., 2008). 

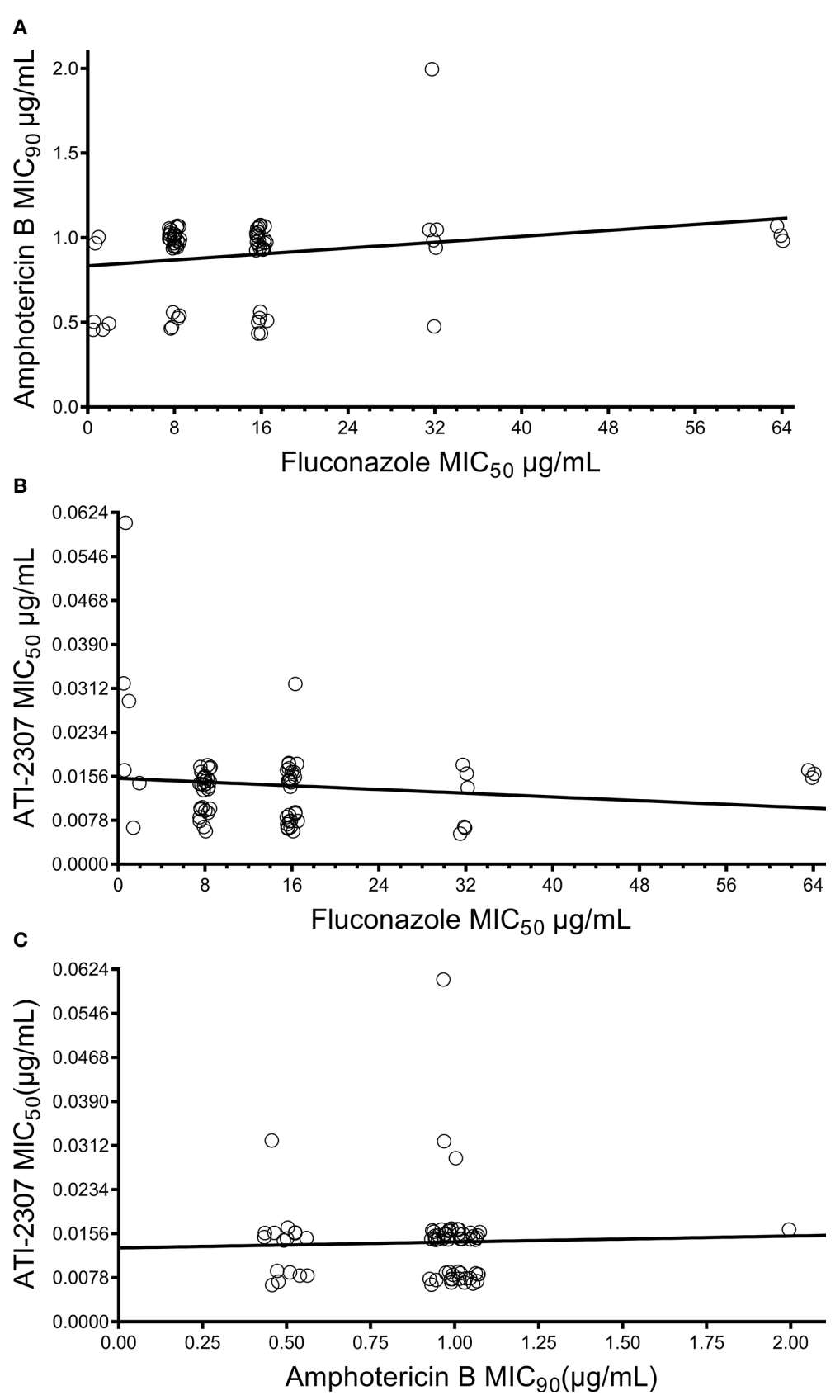

FIGURE 1 | Correlation between fluconazole IC $\mathrm{I}_{50}$, amphotericin B IC 90 , and ATI-2307 IC $\mathrm{C}_{50}$ values for Cryptococcus neoformans clinical isolates. No correlation was observed between (A) fluconazole and amphotericin B susceptibilities (rho $=0.2049, P=0.21$ ), (B) fluconazole and ATI-2307 susceptibilities (rho $=-0.1690, P=$ 0.1650), or (C) amphotericin B and ATI-2307 susceptibilities (rho $=0.0125, P=0.9190)$.

High fluconazole MICs have been documented in several countries throughout the world (Chen et al., 2015; Smith et al., 2015; Naicker et al., 2020). Furthermore, a systematic review of fluconazole MICs by Chesdachai and colleagues shows the median $\mathrm{MIC}_{50}$ is trending upwards, with the potential for current fluconazole dosing guidelines proving inadequate for cryptococcosis in the context of these rising MICs (Chesdachai et al., 2019). While Smith et al. (2015) found no effect of higher fluconazole $\mathrm{IC}_{50}$ in the context of $\mathrm{HIV}$ patients receiving combination therapy with amphotericin B $800 \mathrm{mg} /$ day consolidation therapy in Uganda, a similar study by Nasri and colleagues (2016) with both transplant recipients and individuals with HIV found that patients with high fluconazole $\mathrm{IC}_{50}$ values that received voriconazole or higher-dose fluconazole ( $\geq 800 \mathrm{mg}$ ) 
TABLE 2 | MIC $\mathrm{M}_{50}$ and $\mathrm{MIC}_{90}$ for ATI-2307 and amphotericin B are equivalent across Cryptococcus neoformans clinical isolates with differing fluconazole $\mathrm{IC}_{50}$.

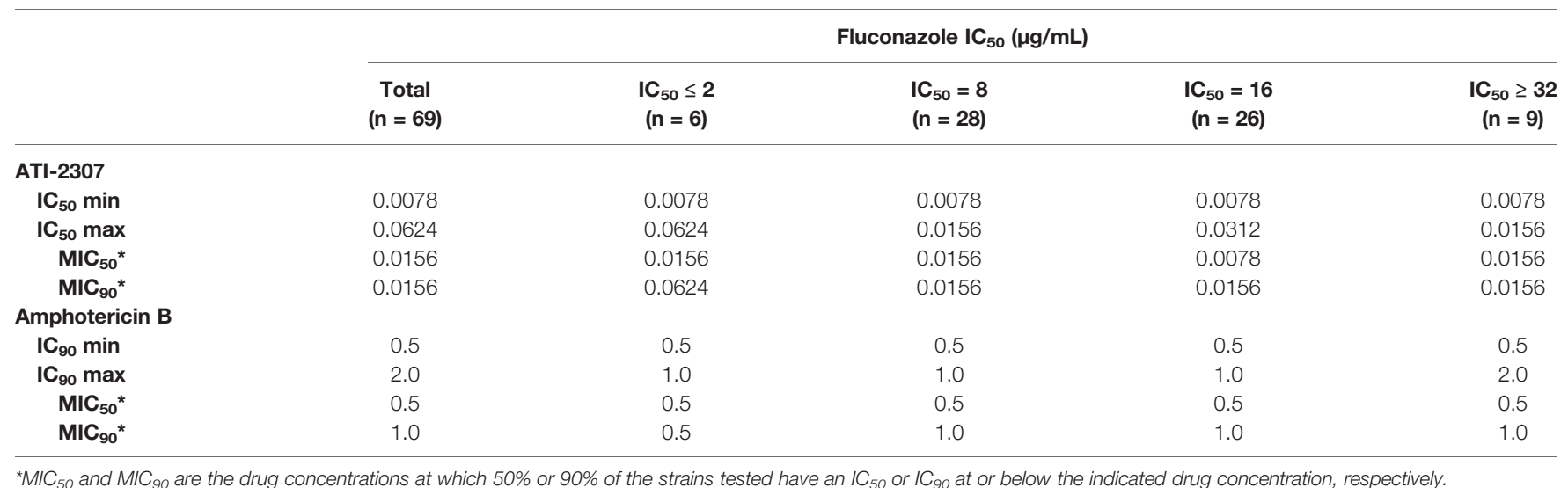

for consolidation therapy were more likely to survive. These results highlight the effectiveness of utilizing drugs with different modes of action in combination therapy approaches to drug treatment, but also the need for new non-azole drugs that can be used during consolidation therapy.

In conclusion, our studies show that ATI-2307 is fully active against clinical isolates of $C$. neoformans with high fluconazole $\mathrm{IC}_{50}$ values. This novel drug has strong potential as a new antifungal therapeutic to increase the arsenal of drugs with differing modes of action that can be explored for use, either in combination or monotherapy approaches, for the treatment of cryptococcal meningitis or antigen positive cryptococcosis.

\section{DATA AVAILABILITY STATEMENT}

The original contributions presented in the study are included in the article. Further inquiries can be directed to the corresponding author.

\section{REFERENCES}

Archibald, L. K., Tuohy, M. J., Wilson, D. A., Nwanyanwu, O., Kazembe, P. N., Tansuphasawadikul, S., et al. (2004). Antifungal Susceptibilities of Cryptococcus neoformans. Emerg. Infect. Dis. 10 (1), 143. doi: 10.3201/eid1001.020779

Arendrup, M. C., Meletiadis, J., Mouton, J. W., Lagrou, K., Hamal, P., Guinea, J., et al. (2017). Method for the Determination of Broth Dilution Minimum Inhibitory Concentrations of Antifungal Agents for Yeasts. EUCAST DEFINITIVE Document E.DEF 7.3.1, European Society of Clinical Microbiology and Infectious Diseases. Available at: https://www.eucast.org/ astoffungi/previous_versions_of_documents/.

Assing, K., Birgens, H., and Arendrup, M. (2003). Cryptococcus neoformans var. neoformans Resistant to Fluconazole in An HIV-Negative Patient With Chronic Lymphocytic Leukemia. Clin. Microbiol. Infect. 9 (5), 441-444. doi: 10.1046/j.1469-0691.2003.00571.x

Bongomin, F., Oladele, R. O., Gago, S., Moore, C. B., and Richardson, M. D. (2018). A Systematic Review of Fluconazole Resistance in Clinical Isolates of Cryptococcus Species. Mycoses 61 (5), 290-297. doi: 10.1111/myc.12747

Boulware, D. R., Meya, D. B., Muzoora, C., Rolfes, M. A., Huppler Hullsiek, K., Musubire, A., et al. (2014). Timing of Antiretroviral Therapy After Diagnosis of Cryptococcal Meningitis. N. Engl. J. Med. 370 (26), 2487-2498. doi: 10.1056/ NEJMoa1312884

\section{AUTHOR CONTRIBUTIONS}

EG, SA, JMY, DB and KN contributed to conception and design of the study. TL, AA, JR, DM, and DB collected the clinical isolates, designed, and performed the clinical trials, EG performed the statistical analysis. EG and KN wrote the first draft of the manuscript. All authors contributed to the article and approved the submitted version.

\section{FUNDING}

This study was supported by Appili Therapeutics, Inc. research award CON000000083519. The funder was not involved in the study design, collection, analysis, interpretation of data, the writing of this article or the decision to submit it for publication. Additional funding to support the collection and analysis of the clinical isolates was supported by NIH grants U01AI089244 and R01NS086312 to DB and R01NS118538 to KN.

Casadevall, A., Spitzer, E. D., Webb, D., and Rinaldi, M. G. (1993). Susceptibilities of Serial Cryptococcus neoformans Isolates From Patients With Recurrent Cryptococcal Meningitis to Amphotericin B and Fluconazole. Antimicrob. Agents Chemother. 37 (6), 1383-1386. doi: 10.1128/aac.37.6.1383

Chen, Y. C., Chang, T. Y., Liu, J. W., Chen, F. J., Chien, C. C., Lee, C. H., et al. (2015). Increasing Trend of Fluconazole-Non-Susceptible Cryptococcus neoformans in Patients With Invasive Cryptococcosis: A 12-Year Longitudinal Study. BMC Infect. Dis. 15, 277. doi: 10.1186/s12879-015-1023-8

Chesdachai, S., Rajasingham, R., Nicol, M. R., Meya, D. B., Bongomin, F., Abassi, M., et al. (2019). Minimum Inhibitory Concentration Distribution of Fluconazole Against Cryptococcus Species and the Fluconazole Exposure Prediction Model. Open Forum Infect. Dis. 6 (10), ofz369. doi: 10.1093/ofid/ofz369

Felton, T., Troke, P. F., and Hope, W. W. (2014). Tissue Penetration of Antifungal Agents. Clin. Microbiol. Rev. 27 (1), 68. doi: 10.1128/CMR.00046-13

Hurtado, J. C., Castillo, P., Fernandes, F., Navarro, M., Lovane, L., Casas, I., et al. (2019). Mortality Due to Cryptococcus neoformans and Cryptococcus gattii in Low-Income Settings: An Autopsy Study. Sci. Rep. 9 (1), 7493. doi: 10.1038/ s41598-019-43941-w

Klepser, M. (2011). The Value of Amphotericin B in the Treatment of Invasive Fungal Infections. J. Crit. Care 26 (2), 225.e1-225.10. doi: 10.1016/j.jcrc.2010.08.005

Mdodo, R., Moser, S. A., Jaoko, W., Baddley, J., Pappas, P., Kempf, M., et al. (2011). Antifungal Susceptibilities of Cryptococcus neoformans Cerebrospinal 
Fluid Isolates From AIDS Patients in Kenya. Mycoses 54 (5), e438-e442. doi: 10.1111/j.1439-0507.2010.01946.x

Meya, D. B., Manabe, Y. C., Castelnuovo, B., Cook, B. A., Elbireer, A. M., Kambugu, A., et al. (2010). Cost-Effectiveness of Serum Cryptococcal Antigen Screening to Prevent Deaths Among HIV-infected Persons With a CD4+ Cell Count $<$ or $=$ 100 Cells/Microl Who Start HIV Therapy in Resource-Limited Settings. Clin. Infect. Dis. 51 (4), 448-455. doi: 10.1086/655143

Mitsuyama, J., Nomura, N., Hashimoto, K., Yamada, E., Nishikawa, H., Kaeriyama, M., et al. (2008). In Vitro and In Vivo Antifungal Activities of T-2307, A Novel Arylamidine. Antimicrob. Agents Chemother. 52 (4), 13181324. doi: 10.1128/aac.01159-07

Molloy, S. F., Kanyama, C., Heyderman, R. S., Loyse, A., Kouanfack, C., Chanda, D., et al. (2018). Antifungal Combinations for Treatment of Cryptococcal Meningitis in Africa. N. Engl. J. Med. 378 (11), 1004-1017. doi: 10.1056/NEJMoa1710922

Naicker, S. D., Mpembe, R. S., Maphanga, T. G., Zulu, T. G., Desanto, D., Wadula, J., et al. (2020). Decreasing Fluconazole Susceptibility of Clinical South African Cryptococcus neoformans Isolates Over a Decade. PloS Negl. Trop. Dis. 14 (3), e0008137. doi: 10.1371/journal.pntd.0008137

Nalintya, E., Meya, D. B., Lofgren, S., Huppler Hullsiek, K., Boulware, D. R., and Rajasingham, R. (2018). A Prospective Evaluation of a Multisite Cryptococcal Screening and Treatment Program in HIV Clinics in Uganda. J. Acquir. Immune Defic. Syndr. 78 (2), 231-238. doi: 10.1097/qai.0000000000001669

Nielsen, K., Cox, G. M., Wang, P., Toffaletti, D. L., Perfect, J. R., and Heitman, J. (2003). Sexual Cycle of Cryptococcus neoformans var. grubii and Virulence of Congenic a and $\alpha$-Isolates. Infect. Immun. 71 (9), 4831-4841. doi: 10.1128/ iai.71.9.4831-4841.2003

Nielsen, K., Vedula, P., Smith, K. D., Meya, D. B., Garvey, E. P., Hoekstra, W. J., et al. (2017). Activity of VT-1129 Against Cryptococcus neoformans Clinical Isolates With High Fluconazole MICs. Med. Mycol. 55 (4), 453-456. doi: 10.1093/mmy/myw089

Nishikawa, H., Fukuda, Y., Mitsuyama, J., Tashiro, M., Tanaka, A., Takazono, T., et al. (2017). In Vitro and In Vivo Antifungal Activities of T-2307, A Novel Arylamidine, Against Cryptococcus gattii: An Emerging Fungal Pathogen. J. Antimicrob. Chemother. 72 (6), 1709-1713. doi: 10.1093/jac/dkx020

Park, B. J., Wannemuehler, K. A., Marston, B. J., Govender, N., Pappas, P. G., and Chiller, T. M. (2009). Estimation of the Current Global Burden of Cryptococcal Meningitis Among Persons Living With HIV/AIDS. AIDS 23 (4), 525-530. doi: 10.1097/QAD.0b013e328322ffac

Perea, S., and Patterson, T. F. (2002). Antifungal Resistance in Pathogenic Fungi. Clin. Infect. Dis. 35 (9), 1073-1080. doi: 10.1086/344058

Pfaller, M. A., Castanheira, M., Diekema, D. J., Messer, S. A., and Jones, R. N. (2011). Wild-Type MIC Distributions and Epidemiologic Cutoff Values for Fluconazole, Posaconazole, and Voriconazole When Testing Cryptococcus neoformans as Determined by the CLSI Broth Microdilution Method. Diagn. Microbiol. Infect. Dis. 71 (3), 252-259. doi: 10.1016/j.diagmicrobio.2011.07.007

Pfaller, M., Zhang, J., Messer, S., Tumberland, M., Mbidde, E., Jessup, C., et al. (1998). Molecular Epidemiology and Antifungal Susceptibility of Cryptococcus neoformans Isolates From Ugandan AIDS Patients. Diagn. Microbiol. Infect. Dis. 32 (3), 191-199. doi: 10.1016/s0732-8893(98)00095-9

Pharkjaksu, S., Chongtrakool, P., Chayakulkeeree, M., Mitrpant, C., Angkasekwinai, P., Bennett, J. E., et al. (2020). Cryptococcus neoformans/ gattii Species Complexes From Pre-HIV Pandemic Era Contain Unusually
High Rate of Non-Wild-Type Isolates for Amphotericin B. Infect. Drug Resist. 13, 673-681. doi: 10.2147/idr.s235473

Rajasingham, R., Smith, R. M., Park, B. J., Jarvis, J. N., Govender, N. P., Chiller, T. M., et al. (2017). Global Burden of Disease of HIV-Associated Cryptococcal Meningitis: An Updated Analysis. Lancet Infect. Dis. 17 (8), 873-881. doi: 10.1016/S1473-3099(17)30243-8

Rhein, J., Huppler Hullsiek, K., Tugume, L., Nuwagira, E., Mpoza, E., Evans, E. E., et al. (2019). Adjunctive Sertraline for HIV-Associated Cryptococcal Meningitis: A Randomised, Placebo-Controlled, Double-Blind Phase 3 Trial. Lancet Infect. Dis. 19 (8), 843-851. doi: 10.1016/s1473-3099(19)30127-6

Rhein, J., Morawski, B. M., Hullsiek, K. H., Nabeta, H. W., Kiggundu, R., Tugume, L., et al. (2016). Efficacy of Adjunctive Sertraline for the Treatment of HIV-Associated Cryptococcal Meningitis: An Open-Label Dose-Ranging Study. Lancet Infect. Dis. 16 (7), 809-818. doi: 10.1016/ s1473-3099(16)00074-8

Roemer, T., and Krysan, D. J. (2014). Antifungal Drug Development: Challenges, Unmet Clinical Needs, and New Approaches. Cold Spring Harb. Perspect. Med. 4 (5), a019703. doi: 10.1101/cshperspect.a019703

Shibata, T., Takahashi, T., Yamada, E., Kimura, A., Nishikawa, H., Hayakawa, H., et al. (2012). T-2307 Causes Collapse of Mitochondrial Membrane Potential in Yeast. Antimicrob. Agents Chemother. 56 (11), 5892-5897. doi: 10.1128/ aac.05954-11

Smith, K. D., Achan, B., Hullsiek, K. H., McDonald, T. R., Okagaki, L. H., Alhadab, A. A., et al. (2015). Increased Antifungal Drug Resistance in Clinical Isolates of Cryptococcus neoformans in Uganda. Antimicrob. Agents Chemother. 59 (12), 7197-7204. doi: 10.1128/aac.01299-15

Vadlapatla, R. K., Patel, M., Paturi, D. K., Pal, D., and Mitra, A. K. (2014). Clinically Relevant Drug-Drug Interactions Between Antiretrovirals and Antifungals. Expert Opin. Drug Metab. Toxicol. 10 (4), 561-580. doi: $10.1517 / 17425255.2014 .883379$

Verweij, P. E., Snelders, E., Kema, G. H., Mellado, E., and Melchers, W. J. (2009). Azole Resistance in Aspergillus fumigatus: A Side-Effect of Environmental Fungicide Use? Lancet Infect. Dis. 9 (12), 789-795. doi: 10.1016/s1473-3099 (09)70265-8

Yamashita, K., Miyazaki, T., Fukuda, Y., Mitsuyama, J., Saijo, T., Shimamura, S., et al. (2019). The Novel Arylamidine T-2307 Selectively Disrupts Yeast Mitochondrial Function by Inhibiting Respiratory Chain Complexes. Antimicrob. Agents Chemother. 63 (8), e00374-19. doi: 10.1128/aac.00374-19

Conflict of Interest: KN received funding from Appili Therapeutics Inc.

The remaining authors declare that the research was conducted in the absence of any commercial or financial relationships that could be construed as a potential conflict of interest.

Copyright (c) 2021 Gerlach, Altamirano, Yoder, Luggya, Akampurira, Meya, Boulware, Rhein and Nielsen. This is an open-access article distributed under the terms of the Creative Commons Attribution License (CC BY). The use, distribution or reproduction in other forums is permitted, provided the original author(s) and the copyright owner(s) are credited and that the original publication in this journal is cited, in accordance with accepted academic practice. No use, distribution or reproduction is permitted which does not comply with these terms. 